\title{
Lien au territoire selon les générations chez les Anicinapek et les Cris
}

\author{
Véronique Landry ${ }^{\mathrm{a}}$, Hugo Asselin ${ }^{\mathrm{b}}$, Carole Lévesque ${ }^{\mathrm{c}}$
}

RÉSUMÉ. L'objectif de cette recherche exploratoire est d'aborder la triple occultation (ruralité, autochtonie et jeunesse) vécue par les jeunes autochtones en milieu rural en documentant les manifestations des trois aspects du lien au territoire (émotionnel, cognitif et fonctionnel) selon les générations dans deux communautés autochtones au Québec. L'appartenance au territoire (aspect émotionnel) est présente à tous les âges, mais selon une hiérarchie différente entre forêt, communauté et ville. Toutes les générations fréquentent la forêt, mais à des moments différents et pour y pratiquer des activités différentes. Le chez-soi des jeunes est la communauté, tandis que celui des aînés, la forêt. L'aspect cognitif se reflète par la perception de menace face à la dégradation du territoire, particulièrement chez les aînés. Quelques jeunes ont un faible lien au territoire, probablement parce que cette période de la vie est caractérisée par une quête identitaire. Toutes les générations pratiquent des activités traditionnelles (aspect fonctionnel), mais de façon différente.

\begin{abstract}
The objective of this exploratory research is to address the triple occultation (rurality, indigeneity, and youth) experienced by young indigenous people in rural areas, by documenting how the three aspects of the link to the land (emotional, cognitive, and functional) are displayed by different generations in two indigenous communities in Quebec. There is a sense of belonging to the land (emotional aspect) at all ages, although the forest, community and city have different levels of importance. All generations spend time in the forest, but at different times and to practise different activities. The youth identify their community as home, whereas for the elderly it is the forest. The cognitive aspect is reflected in the perception of a threat associated to the degradation of the land, particularly among the elderly people. Some young people are weakly linked to the land, probably because this period of life is characterized by a quest for identity. All the generations practise traditional activities (functional aspect), but in different ways.
\end{abstract}

\section{Introduction}

La ruralité est souvent abordée du point de vue des urbains et, par conséquent, «les ruraux n'ont pas la parole » (Jean, 2017, p. 177). Cela est d'autant plus vrai dans le cas des peuples autochtones, qui se considèrent comme "les éternels oubliés" (Kistabish, Kistabish, Mapachee et Rankin, 2011, p. 67). Et encore plus pour les jeunes autochtones, dont la parole soufre d'un « manque de documentation » en raison de l'« attention coutumière [portée] aux personnes âgées » (Bousquet, 2005, p. 7). Considérant que, pour les Autochtones, le territoire est «matrice de culture » (Martin et Girard, 2009), l'objectif de cette recherche exploratoire est d'aborder la triple occultation associée à la ruralité, à l'autochtonie et à la jeunesse en documentant les manifestations du lien au territoire selon les générations dans deux communautés autochtones au Québec.

Le lien au territoire découle de l'importance et de la signification qu'y accorde une personne ou un groupe (Stedman, 2003). Il se compose de trois aspects : émotionnel (l'attachement affectif au territoire), cognitif (les croyances et perceptions du territoire et le fait de s'y identifier) et fonctionnel (la capacité du territoire à répondre aux besoins) (Anton et Lawrence, 2014; Jorgensen et Stedman 2006; Junot, Paquet et Fenouillet, 2018).

\footnotetext{
a Doctorante, Institut de recherche sur les forêts, Université du Québec en Abitibi-Témiscamingue

b Professeur titulaire et directeur, École d'études autochtones, Université du Québec en Abitibi-Témiscamingue

${ }^{\text {C }}$ Professeure titulaire, Institut national de la recherche scientifique, Centre Urbanisation Culture Société
} 
L'attachement représente l'aspect émotionnel du lien au territoire. Selon Low et Altman (1992), il implique non seulement des connaissances et des croyances relatives au territoire, mais également des émotions et des sentiments qui permettent de créer un lien affectif. Ensuite, l'aspect cognitif du lien au territoire relève de son rôle dans la construction de l'identité (Junot et collab., 2018; Scannell et Gifford, 2010). L'identité n'est ni statique ni définitive, et elle se définit par un processus continu de retour sur soi-même. Il en va de même pour le lien au territoire, qui est continuellement « renégocié ", individuellement et collectivement (Berkes, 2014; Cheng, Kruger et Daniels, 2003). Enfin, l'aspect fonctionnel du lien au territoire découle de sa capacité à satisfaire aux besoins (Jorgensen et Stedman, 2006; Tidball et Stedman, 2013).

\section{Méthodologie}

L'histoire de vie, l'âge, le parcours géographique et la culture sont autant d'éléments qui influencent le lien au territoire (Cheng et collab., 2003). Par conséquent, le lien au territoire se vit différemment selon la génération (Bousquet, 2002; Guérin-Pace, 2006). Dans ce travail, quatre générations sont distinguées sur la base des travaux de Bousquet (2002) avec les Anicinapek (Algonquins) de Pikogan, en Abitibi-Témiscamingue. Les générations réfèrent non seulement à l'âge des personnes, mais également au fait qu'elles sont nées dans un espace historico-social distinct et qu'elles partagent un même vécu.

D'abord, les membres de la génération plus âgée, "les aînés ${ }^{1}$ » (61 ans et plus au moment de la collecte de données en 2013-2014), ont connu la sédentarisation progressive après leur naissance « dans le bois». Ils ne parlent souvent que leur langue autochtone et vivent une certaine nostalgie de la vie en forêt. Pour plusieurs, la communauté est vue comme une prison et associée à des métaphores négatives qui évoquent la domination (Bousquet, 2002), tandis que la forêt a une charge positive (Saint-Arnaud, Asselin, Dubé, Croteau et Papatie, 2009).

La génération suivante (46-60 ans) a vécu les « pensionnats indiens », qui ont principalement été mis en place des années 1950 aux années 1970. Sous le prétexte de l'éducation, les pensionnats étaient davantage des centres d'endoctrinement culturel.
La génération « post-pensionnat» (31-45 ans) est née en communauté ou en ville et considère ce lieu comme sa maison (Basile, Asselin et Martin, 2017; Guérin-Pace, 2006). Sa fréquentation de la forêt est sporadique, mais implique tout de même une certaine valorisation des activités traditionnelles (p. ex., chasse, pêche, trappe et cueillette).

Enfin, les membres de la jeune génération (18-30 ans) sont nés en ville, ont vécu la majorité de leur vie en communauté, fréquentent peu la forêt et ont une faible connaissance des langues autochtones.

Un questionnaire comprenant 53 questions à choix multiples ou à développement court a été administré dans la communauté crie de Waswanipi et dans la communauté anicinape de Pikogan afin d'explorer le lien au territoire selon les générations. Les répondants étaient invités à commenter les questions et à préciser leurs réponses, ce qui a permis d'enrichir le corpus et de mieux contextualiser les résultats.

Waswanipi est la plus méridionale des 9 communautés cries au Québec. Eeyou Istchee - le territoire cri - a fait l'objet de la signature d'un traité moderne en 1975 : la Convention de la Baie-James et du Nord québécois (CBJNQ). L'entente précise les modalités d'utilisation et de gouvernance du territoire. Elle a été complétée en 2002 par la Paix des Braves, puis en 2012 par l'Entente sur la gouvernance dans le territoire d'Eeyou Istchee BaieJames. Le territoire traditionnel de la communauté de Waswanipi, qui compte un peu plus de 2200 membres, est situé dans la forêt boréale, sujette à l'exploitation forestière.

Pikogan est la plus septentrionale des 9 communautés anicinapek au Québec. Ses quelque 1100 membres utilisent le Nitakinan - le territoire traditionnel - situé dans la forêt boréale commerciale. Contrairement aux Cris, les Anicinapek n'ont à ce jour pas encore signé de traité moderne avec les gouvernements. Les Cris et les Anicinapek étaient autrefois semi-nomades et organisaient leurs déplacements en fonction des activités saisonnières de chasse, de trappe, de pêche et de cueillette. Encore aujourd'hui, ces activités constituent une part importante de leur mode de vie et de leur identité (Niezen, 2016; Saint-Arnaud et collab., 2009; Tobias et Richmond, 2014). 
Le questionnaire a été élaboré à partir des travaux de l'enquête provinciale de l'Alliance de recherche ODENA (www.odena.ca), qui a fait un portrait statistique des Autochtones vivant en milieu urbain, ainsi que des travaux de Bousquet (2002) et de Saint-Arnaud et ses collaborateurs (2009), qui ont décrit en détail les représentations du territoire dans deux communautés anicinapek. Le questionnaire a été administré en présentiel par Véronique Landry, assistée de Maggie Etapp, cochercheuse autochtone. Des organismes partenaires (Centre d'amitié autochtone de Val-d'Or, Conseil de bande de Pikogan, Autorité forestière de Waswanipi et Pavillon des Premiers-Peuples de l'UQAT à Val-d'Or) ont aidé au recrutement des participants. L'effet «boule de neige » (Gamborg, Parsons, Puri et Sandøe, 2012) a permis d'atteindre la saturation de l'information, c'est-à-dire un point où des entrevues additionnelles ne permettaient pas d'obtenir d'information supplémentaire.
Les critères d'inclusion des participants étaient le lieu de résidence et l'âge. Les 54 participants ont été classés selon leur génération et leur communauté d'appartenance (Tableau 1). Dans la section Résultats, les participants sont identifiés par un code pour protéger leur anonymat : une lettre représentant la communauté $(\mathrm{W} / \mathrm{P})$, suivie d'un chiffre par individu, puis d'un tiret et du code de génération. La comparaison des distributions de fréquence des réponses aux questions a permis de relever des différences (bien que non statistiquement représentatives) entre les quatre générations (13 à 15 répondants par génération). Ces différences ont été analysées en regard des trois aspects du lien au territoire : émotionnel, cognitif et fonctionnel (Jorgensen et Stedman, 2006).

Le projet a été approuvé par le Comité d'éthique de la recherche de l'Université du Québec en Abitibi-Témiscamingue (UQAT) et a reçu l'appui des conseils de bande des deux communautés participantes.

\begin{tabular}{lccc}
\hline & $\begin{array}{c}\text { Code de } \\
\text { génération }\end{array}$ & Waswanipi & Pikogan \\
\hline Aînés (61 ans et plus) & 4 & 7 & 6 \\
Pensionnat (46-60 ans) & 3 & 6 & 7 \\
Post-pensionnat (31-45 ans) & 2 & 9 & 6 \\
Jeunes (18-30 ans) & 1 & 6 & 7 \\
\hline
\end{tabular}

Tableau 1 - Répartition des répondants, selon la génération et la communauté

\section{Résultats}

De façon générale, il n’y a pas de différence marquée entre les répondants selon le genre. Les résultats sont donc présentés de façon globale, sauf pour certains aspects précis, notamment le fait d'être responsable d'un terrain de trappe familial, où des distinctions sont faites. De la même façon, il n'y a pas de différence marquée entre les répondants selon leur communauté d'appartenance. Les résultats sont par conséquent présentés globalement, mais sont parfois nuancés.

\subsection{Aspect émotionnel}

Cinq variables ont permis d'évaluer l'aspect émotionnel du lien au territoire : 1) le lieu que les répondants considèrent comme leur chez-soi; 2) les raisons qui justifient ce choix; 3) le lieu d'enfance; 4) la fréquentation actuelle de la forêt; et 5) le sentiment d'appartenance au lieu de résidence.

Dans les deux communautés, les 18-30 ans identifient davantage leur chez-soi comme étant la communauté, tandis que les plus âgés (61 ans et plus) indiquent plutôt la forêt (Figure 1). Les adultes de 31-60 ans sont partagés entre la communauté et la forêt, mais penchent tout de même plus pour la communauté. 


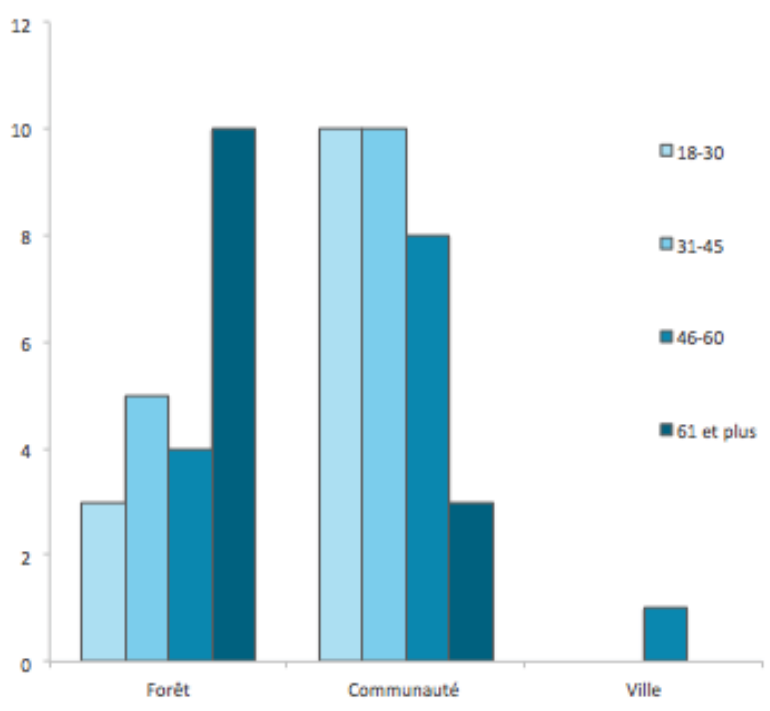

Figure 1 - Lieu identifié comme leur chez-soi par les répondants, selon la génération

Certains répondants habitent plusieurs endroits et partagent leur temps entre la communauté et la forêt, mais aussi la ville. Par exemple, W6-2 habite temporairement à deux endroits, le temps de terminer des études en ville. Sa maison dans la communauté est identifiée comme son chez-soi. Plusieurs personnes partagent leur temps entre le terrain de trappe et la communauté, comme W13-4, dont le conjoint est décédé, qui vit sur son terrain de trappe avec des amis, tout en passant aussi du temps dans la communauté. Certains maitres de trappe identifient leur terrain de trappe comme leur chez-soi.

Par ailleurs, même si une personne vit à un endroit, elle ne l'identifie pas nécessairement comme son chez-soi. Par exemple, W2-1 estime que la vie en forêt est son mode de vie aujourd'hui et que son chez-soi est davantage la forêt que la communauté. Dans sa réalité actuelle, il ne fréquente toutefois la forêt qu'à l'occasion et prévoit d'aller habiter en ville afin de poursuivre des études, pour revenir ensuite habiter son terrain de trappe.

L'identification d'un lieu en tant que chez-soi est généralement liée aux émotions ou au fait que l'endroit est le lieu d'origine («c'est là d'où je viens », «ma famille vient de là», "présence de mes ancêtres »). L'aspect émotionnel est souvent exprimé en associant le territoire à la famille, et ce, pour toutes les générations. "Mon territoire de chasse, c'est de l'affection familiale, c'est mon devoir, notre responsabilité.» (P24-3) «Mon lien au territoire, je le décrirais comme familial. Là-bas, dans le bois, c'est la famille. C'est rassembleur, c'est la proximité. » (W3-3)

Le chez-soi est également lié à un aspect fonctionnel (« ma maison est ici», «mon travail est ici »). Certains répondants précisent que c'est un lieu de bien-être. Les jeunes (18-30 ans) nomment plus de raisons émotives, tandis que les plus âgés (46 ans et plus) ont plutôt tendance à nommer les raisons fonctionnelles ou le bien-être. Ainsi, P8-3 affirme : « La vie dans le bois, pour moi, c'est la meilleure façon de vivre; tout est là. Ce n'est pas payant économiquement, mais c'est payant à l'intérieur de moi. Le bien-être est là. Moi, ce que je connais, c'est le bois; je l'ai dans le sang. »

Lorsque les répondants doivent identifier à quel(s) endroit(s) ils ont vécu leur enfance (de 0 à 18 ans), les deux générations plus jeunes identifient majoritairement la ville ou la communauté, tandis que les deux générations plus âgées mentionnent majoritairement la forêt (Figure 2). À Waswanipi, la moitié des jeunes (18-30 ans) identifie le premier lieu d'enfance comme étant la forêt, tandis que l'autre moitié identifie plutôt la communauté. De leur côté, la grande majorité des ainés disent que la forêt est leur lieu d'enfance. Les répondants de 46-60 ans sont partagés entre la forêt, la communauté et la ville. À Pikogan, les résultats diffèrent pour les générations des 18-30 ans et des 31-45 ans, dont la majorité identifie la communauté ou la ville comme lieu d'enfance, alors que les aînés mentionnent tous la forêt. Dans les deux communautés, le deuxième lieu d'enfance le plus fréquent des répondants de 46 ans et plus est le pensionnat. 


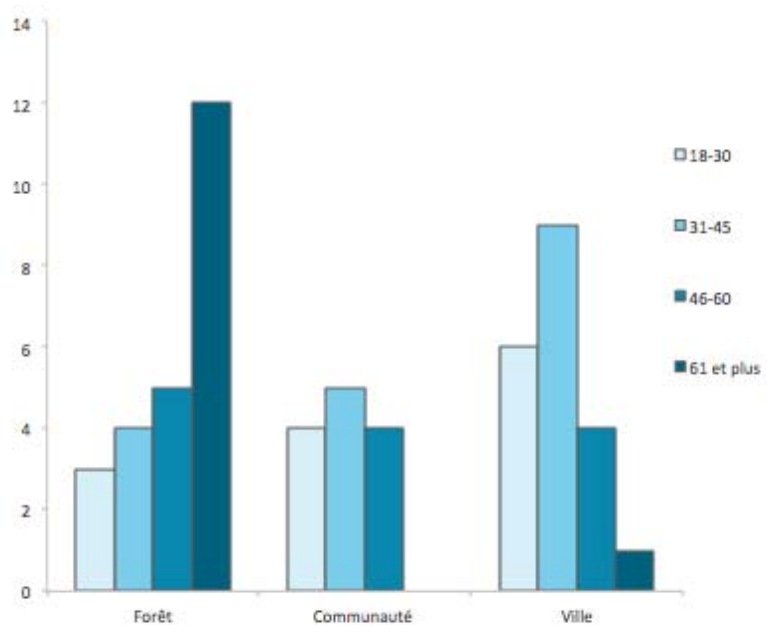

Figure 2 - Premier lieu d'enfance, selon la génération

Seulement deux jeunes (18-30 ans) disent ne pas fréquenter la forêt. La fréquentation de la forêt varie selon les réalités de chacun: les travailleurs peuvent y aller les fins de semaine, d'autres peuvent y passer 10 mois par année et n'en sortir que pour se ravitailler. Tous les répondants indiquent participer aux semaines culturelles à Pikogan et au Moose Break et au Goose Break à Waswanipi, qui permettent aux familles de se rassembler en forêt. Un répondant mentionne toutefois que la fréquentation de la forêt ne devrait pas se limiter à de courtes périodes circonscrites: "Pendant les semaines culturelles, c'est là que le monde va dans le bois. Mais pourquoi attendre? Les traditions et la langue se perdent, l'enseignement de la culture manque. » (P16-4)

Plusieurs répondants relatent de beaux souvenirs liés à des expériences vécues (expéditions hivernales en raquettes ou estivales en canot), à des moments ou à des lieux précis : «Sur le territoire de mon père, il y a un endroit où je retourne toujours, un endroit où on avait un camp qui a été détruit à la suite du divorce de mes parents. C'est la paix, ça me ramène de beaux souvenirs, je m'y sens bien. » (P23-1)

Les répondants de toutes les générations montrent un sentiment d'appartenance envers leur lieu de résidence actuel. Quelques-uns nuancent leurs propos en précisant que leur sentiment d'appartenance est encore plus fort envers leur terrain de trappe. Plus les répondants sont âgés, plus ils manifestent une préférence pour la forêt.

\subsection{Aspect cognitif}

Pour comparer l'aspect cognitif du lien au territoire entre les générations, six variables sont utilisées : 1) le lien au territoire; 2) le lieu de naissance; 3) les enseignements traditionnels reçus (ou non); 4) la langue parlée; 5) les éléments mis de l'avant pour se décrire; et 6) le fait d'avoir habité en ville ou non.

Tous les répondants ont un lien «fort» au territoire, sauf six des 18-30 ans, qui le décrivent comme étant «faible» (4) ou qui disent ne pas en avoir (2). Certains relatent avoir été (ou être) dans une période où ils bâtissent leur vie, se détachant momentanément de ce qu'ils connaissent, mais sachant que c'est pour mieux y revenir plus tard. "Quand on était enfant, on était plus souvent dans le bois. Avec l'adolescence, ça m'intéressait moins. Ça revient. » (P17-1) «À 35-40 ans, il y a comme un retour de la culture et les gens veulent retourner dans le bois. » (P18-4).

Pour toutes les générations, la vie en forêt est encore associée au présent : "C'est quelque chose que j'entretiens encore, c'est la belle vie! » (P23-1) «Je me sens responsable de prendre soin de ce qu'on m'a laissé et de poursuivre les pratiques de mes grands-parents. » (P3-2) Un jeune (18-30 ans) associe toutefois la vie en forêt au passé : « La vie dans le bois, c'est une manière de me ressourcer, mais c'est le bon vieux temps pour moi. Pour nous les jeunes, pour certains, c'est ennuyant, la vie dans le bois. Avec les technologies, maintenant, on a moins d'intérêt. » (P11-1) Un répondant souligne que les savoirs et pratiques associés à la 
vie en forêt sont dynamiques : «On s'adapte, mais on ne perd pas l'identité. » (W19-2)

Alors que presque la moitié des répondants de Waswanipi indiquent être nés en forêt, seule une minorité des répondants de Pikogan répond la même chose; la plupart disent plutôt être nés à Amos, la ville voisine. A Waswanipi, les 46 ans et plus sont majoritairement nés en forêt, tandis que les 18-45 ans, en ville (Chibougamau, Matagami, Val-d'Or). Aucun répondant, ni de Waswanipi ni de Pikogan, n'a dit être né dans sa communauté.

Tous les répondants, toutes générations confondues, ont reçu des enseignements par des membres de leur famille ou par des aînés, et tous ont transmis des enseignements.

La première langue parlée est, dans une forte proportion, l'anicinapemowin (Pikogan) ou l'iyniw- ayamiwin (Waswanipi) (Figure 3). Toutefois, à Pikogan, peu de jeunes (18-45 ans) indiquent l'anicinapemowin comme première langue.

Certains répondants établissent un lien direct entre la locution des langues autochtones et la fréquentation du territoire forestier : «On parle plus algonquin dans le bois. C'est vrai que notre langue est une langue qui est très imagée et que ces images font plus référence à ce qui est dans le bois qu'à ce qui est en ville. » (P18-4)

Les thèmes les plus populaires utilisés par les répondants pour se décrire sont: famille/amis et territoire/culture (Figure 4).

Plus de la moitié des répondants ont déjà habité en ville (56\%). Parmi ceux-ci, la plupart sont âgés de 31 à 60 ans (20 sur 30). Peu des 18-30 ans ont déjà habité en ville (3 sur 13).

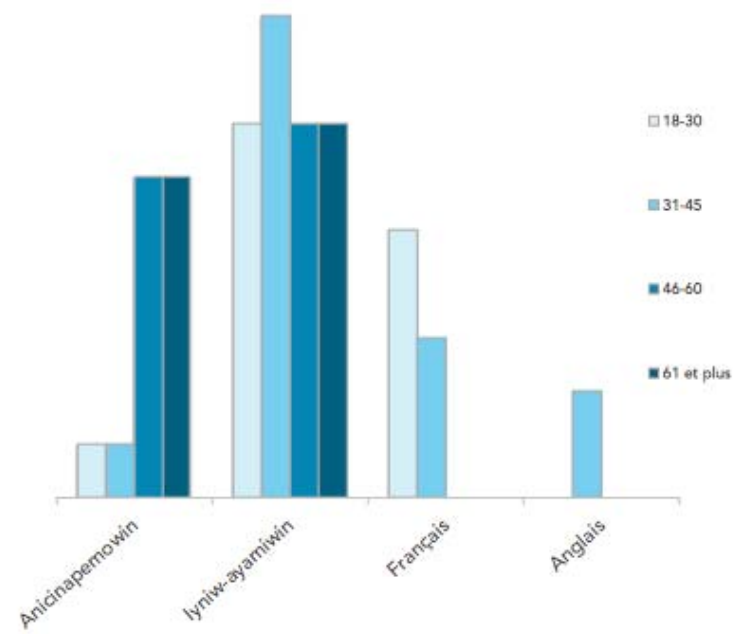

Figure 3 - Langue maternelle parlée, selon la génération

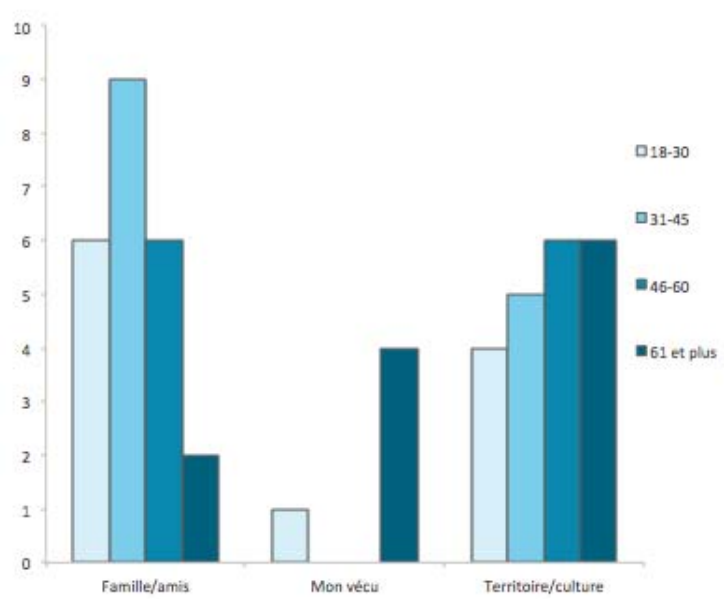

Figure 4 - Principal terme utilisé par les répondants pour se décrire, selon la génération 


\subsection{Aspect fonctionnel}

Pour comparer l'aspect fonctionnel du lien au territoire selon les générations, six variables sont utilisées : 1) l'occupation dans la vie; 2) la responsabilité ou non d'un terrain de trappe; 3) la facilité d'accès à une voiture pour aller en forêt; 4) la pratique ou non d'activités traditionnelles en forêt; 5) envisager ou non de déménager; et 6) le partage des produits des activités traditionnelles.

Les répondants de 61 ans et plus sont retraités (5/13) ou reçoivent des prestations pour les personnes vivant de la chasse ou de la trappe (6/13). Les 46-60 ans sont majoritairement des travailleurs (11/13). Les 31-45 ans reçoivent des prestations pour chasseurs/trappeurs (6/15) ou travaillent (9/15). Quant aux 18-30 ans, la plupart travaillent ou sont aux études (7/13), tandis que les autres vivent de la prestation pour chasseurs/trappeurs (5/13).

Pour les 18 à 30 ans, la responsabilité d'un terrain de trappe revient en majorité à un proche; seulement deux répondants de cette génération disent être responsables d'un terrain de trappe (Figure 5). Chez les 31-45 ans, une faible proportion est responsable d'un terrain de trappe, alors que la majorité fréquente celui d'un proche. Chez les 46-60 ans, $43 \%$ sont responsables d'un terrain de trappe. Enfin, la majorité des 61 ans et plus sont responsables d'un terrain de trappe. Près de deux fois plus d'hommes que de femmes sont responsables d'un terrain de trappe.

Certains répondants mentionnent faire face à des contraintes logistiques d'accès au territoire forestier : «Si j'avais une voiture, j'y irais plus souvent. Si j'avais un camp aussi, ça aiderait. » (P22-2) Certains arrivent quand même à se débrouiller pour se rendre en forêt (autostop, covoiturage, emprunt de voiture). D'autres problèmes d'accès au territoire forestier sont mentionnés : la grande distance entre la communauté et le terrain de trappe, les chemins forestiers fermés ou endommagés et le manque de moyens financiers. Des répondants disent faire face à des obstacles qui relèvent de leur santé.

Hormis deux jeunes (18-30 ans), tous les répondants pratiquent des activités traditionnelles, et ce, à différents moments de l'année (p. ex., chasse, pêche, préparation de la nourriture traditionnelle, cérémonies, médecine, purification à la sauge, fumage de poisson et coupe de bois). Plusieurs répondants soulignent l'adaptation et l'évolution des pratiques : «Mon mode de vie dans le bois, c'est ça encore aujourd'hui, mais ç'a évolué. Maintenant, il faut que ce soit accessible et que ce soit bien équipé. » (P4-4)

Quant à l'intention de déménager, les répondants qui considèrent avoir un rôle important à jouer chez eux ont tendance à souhaiter rester dans la communauté : «Avant, je voulais partir de Pikogan parce qu'il y avait trop de drogue et d'alcool, mais maintenant, je veux rester parce que ma communauté a besoin de moi.» (P23-1) La volonté de déménager en forêt est plus forte chez les 46-60 ans (Figure 6) et la raison la plus souvent mentionnée est la santé et le bien-être. Les répondants de 61 ans et plus envisagent peu de déménager; pour eux, la santé et le bien-être sont également la raison la plus évoquée, mais pour rester. Les jeunes (18-30 ans) souhaitent surtout déménager en ville, tandis que les 31-45 ans donnent des réponses variées.

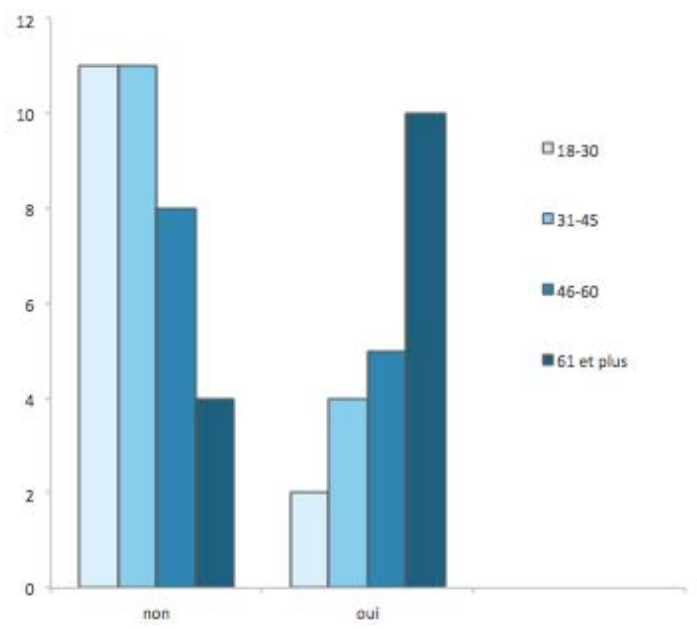

Figure 5 - Responsabilité d'un terrain de trappe, selon la génération 


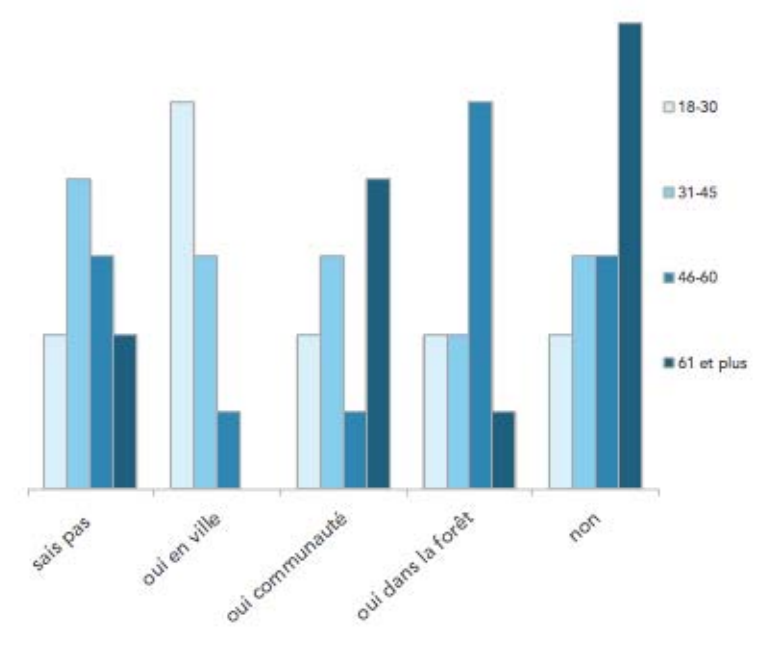

Figure 6 - Intention de déménager, selon la génération

Les raisons motivant l'envie de déménager sont réparties entre la santé, le bien-être, la recherche d'un logement plus adéquat ou la poursuite des études (Figure 7). Certains parents (particulièrement à Waswanipi, communauté plus loin que Pikogan des centres urbains) affirment souhaiter déménager, du moins temporairement, afin d'offrir plus d'opportunités à leur famille, à leurs enfants.

Tous les répondants disent partager les fruits de leurs prélèvements sur le territoire forestier, que ce soit de la viande, des fruits, du poisson ou des produits servant à l'artisanat. Ils confirment aussi, en grande majorité, que les autres membres de la communauté partagent avec eux. Toutefois, certains affirment que les choses ont changé : "Les gens s'attendent à se faire payer. Ce n'est pas bien. Avant, c'était mieux. » (W16-4) «Certains viennent souvent quêter parce qu'ils savent que j'habite sur mon territoire de trappe. » (W26-4) À Waswanipi, un service de distribution de repas traditionnels est offert aux aînés trois fois par semaine, grâce à des contributions des membres de la communauté. Pour les aînés, il serait hors de question de payer pour ce service : «La nourriture traditionnelle a toujours été gratuite. Pourquoi ça changerait? » (W17-4)

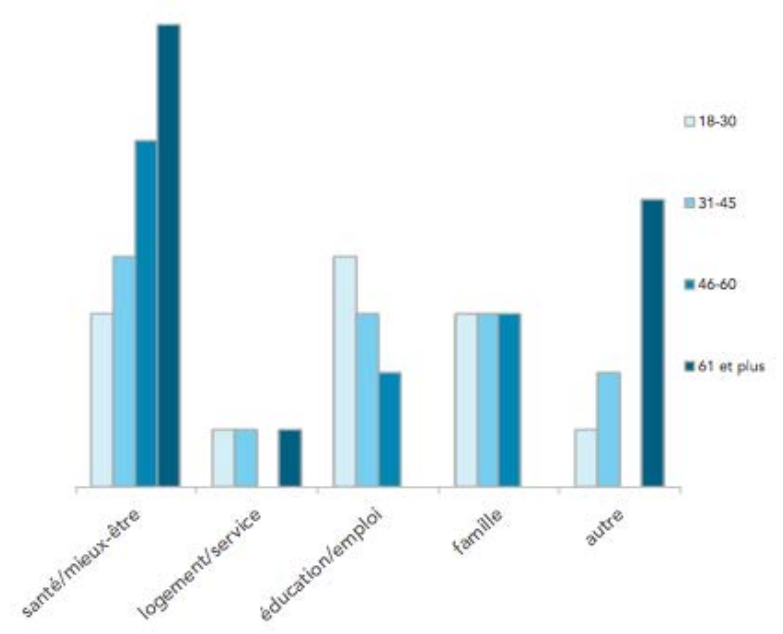

Figure 7 - Raisons de vouloir déménager, selon la génération 


\section{Discussion}

Ce projet a permis de montrer que le lien au territoire se manifeste différemment selon les générations dans deux communautés autochtones au Québec, et ce, en fonction des aspects émotionnel, cognitif et fonctionnel.

\subsection{Aspect émotionnel}

Le sentiment d'appartenance au territoire est présent chez tous les groupes d'âge, mais selon des hiérarchies différentes. Toutes les générations fréquentent la forêt, mais selon des périodes différentes et pour y pratiquer des activités différentes.

Les aînés ont vécu l'implantation des réserves comme une contrainte à leur liberté. Ils sont pour la plupart nés en forêt et y ont vécu une bonne partie de leur enfance (Bousquet, 2002). Toutefois, ils ont un sentiment d'appartenance envers la communauté où habitent famille et amis. L'époque des pensionnats, deuxième lieu d'enfance de tous les répondants de plus de 45 ans, a provoqué une « cassure » dans la vie (Basile et collab., 2017; Wilson, Rosenberg et Abonyi, 2011) dans le lien au territoire et dans l'identité culturelle de plusieurs familles et communautés.

Le soutien de la famille et des amis est déterminant dans la santé holistique des peuples autochtones (Big-Canoe et Richmond, 2014; Landry, Asselin et Lévesque, 2019). Dans leur étude sur la santé autochtone en fonction de l'âge, Wilson et ses collaborateurs (2011) ont relevé que les jeunes ont des niveaux plus élevés de tous les types de soutien social, ce qui a une incidence significative sur le fait de se déclarer en bonne santé. En effet, l'intensité de l'investissement dans un réseau social attaché à un lieu a un effet déterminant sur l'attachement (McMillan et Chavis, 1986; Stedman, 2003). Les 18-45 ans ont passé plus de temps en ville et dans la communauté durant leur enfance, ce qui a un effet sur leur attachement à ces lieux. Les jeunes ont vécu leur enfance dans la communauté et y trouvent des repères géographiques, temporels et émotifs (Anton et Lawrence, 2014). Cette appartenance à la communauté demeure prépondérante, y compris pour la nouvelle génération née en ville (Lévesque et Cloutier, 2013).

Tous les répondants fréquentent la forêt sur de plus ou moins longues périodes et plus ou moins fréquemment, de façon modulée selon leur contexte et la période de vie dans laquelle ils se situent (p. ex., étudiants, travailleurs, retraités). Le fait d'avoir vécu des moments marquants en certains lieux contribue à développer ou à renforcer le lien au territoire : le fait d'y avoir vécu des émotions, d'avoir développé des connaissances et des croyances liées à ce lieu, ainsi que des comportements et des actions qui y font référence (Jorgensen et Stedman, 2006; Low et Altman, 1992). Les activités traditionnelles se poursuivent, mais tiennent compte des nouvelles réalités et s'adaptent aux nouveaux besoins (Asselin, 2015). Par exemple, les déplacements sur le territoire forestier qui se faisaient autrefois à pied ou en raquettes se font désormais en véhicule motorisé; la communication à distance, qui se faisait autrefois par émetteur radio, se fait désormais par téléphone satellitaire ou cellulaire et par les réseaux sociaux (Lemelin et collab. 2010).

\subsection{Aspect cognitif}

Certaines différences intergénérationnelles s'expriment dans l'aspect cognitif du lien au territoire. Seuls quelques répondants parmi les plus jeunes disent avoir un faible lien au territoire, voire aucun lien. Ce résultat peut s'expliquer par le fait que cette période de la vie est souvent caractérisée par une quête identitaire qui se concrétise par de l'exploration personnelle et professionnelle. Dans une conception développementale et psychosociale, l'identité est considérée comme une synthèse d'éléments du passé (histoire personnelle), de caractéristiques du présent (besoins, traits de personnalité) et d'attentes envers le futur (Lannegrand-Willems, 2012). Pour les aînés, bien que le lien au territoire soit fort émotivement et spirituellement, l'état de santé de certains ne leur permet pas d'aller en forêt aussi souvent que souhaité. Ils sont par conséquent limités dans leur rôle de transmission des savoirs, laquelle se fait majoritairement sur le territoire (Asselin, 2015; Barreau, Ibarra, Wyndham, Rojas et Kozak, 2016; Basile et collab., 2017; Hernández, Hidalgo, Salazar-Laplace et Hess, 2007). Les semaines culturelles (à Pikogan) ainsi que le Moose Break et le Goose Break (à Waswanipi) ont été instaurés vers la fin des années 1970 pour assurer à tous les membres des communautés des moments de transmission des savoirs en forêt (Basile et collab., 2017). Ces rassemblements et les cérémonies qui y sont pratiquées sont un moyen de maintenir l'identité culturelle (Tobias et Richmond, 2014). 
L'identité collective joue un rôle déterminant dans le lien au territoire puisque c'est la partie de la conception de soi qui découle de l'appartenance à un groupe social (Ashmore, Deaux et McLaughlinVolpe, 2004). Un des facteurs réduisant l'identité culturelle est la dépossession environnementale, qui entraîne une modification du sens et de la fonction des relations sociales (Big-Canoe et Richmond, 2014; Fuentes, Asselin, Bélisle et Labra, 2020; Richmond, 2015). La dépossession environnementale fait référence aux processus par lesquels l'accès des peuples autochtones à leur territoire est réduit ou interrompu (Richmond et Ross, 2009). Elle se traduit par une perte de territoire de manière directe et indirecte: extraction des ressources naturelles, changements climatiques, projets de développement, etc. (Berry et collab., 2010). La dépossession environnementale serait également à la source de l'érosion des liens entre les générations (Richmond, 2015). Les aînés sont inquiets et craignent pour la poursuite des activités traditionnelles et, par conséquent, pour la continuité culturelle et l'identité, intrinsèquement liées au territoire forestier (Germain, 2012; Richmond, 2015).

Le lieu de naissance permet de se définir en tant qu'Autochtone et le fait de naitre en forêt est un marqueur spatiotemporel important qui assure un lien avec le territoire, qui contribue à l'identité et qui garantit l'attribution de droits de chasse et de pêche pour la vie (Basile et collab., 2017; Dietsch et collab., 2011). Le territoire façonne les aspects culturels, spirituels, émotionnels, physiques et sociaux de la vie (Wilson, 2003). Les hôpitaux et autres établissements dans lesquels sont emmenées les personnes nécessitant des soins de début ou de fin de vie ne sont pas des lieux culturellement pertinents (McGrath, 2007).

Les termes les plus utilisés par les répondants pour se décrire sont famille/amis et territoire/culture. Les origines, notamment familiales, occupent une place importante dans la définition de l'identité (Ramos, 2006). Des différences entre les communautés sont observées quant à la proportion de répondants affirmant être nés en forêt. Des éléments historiques permettent de contextualiser ce résultat. La communauté de Pikogan a été créée en 1958, après quoi beaucoup moins d'enfants ont vécu en forêt. La communauté de Waswanipi a été créée 20 ans plus tard, en 1978.
Puisque les ainés parlent surtout l'anicinapemowin ou l'iyniw-ayamiwin et que les jeunes ne parlent souvent que le français ou l'anglais, les échanges sont parfois difficiles. Les jeunes passent souvent plus de temps à l'école et moins de temps dehors avec les aînés (Barreau et collab., 2016; Basile et collab., 2017). Plusieurs actions sont posées afin de contrer cette dévitalisation de la langue et de favoriser la solidarité intergénérationnelle (Viscogliosi et collab., sous presse).

Pendant longtemps, la perception dominante était que les Autochtones qui s'établissaient en ville perdaient leur identité (Kermoal et Lévesque, 2010). Or, l'identité demeure - et se renforce même, pour certains (Desbiens, Lévesque et Comat, 2016). Le fait de déménager en ville amène l'individu à rechercher une cohérence autour de son histoire et de ses appartenances ou encore à faire ou refaire un «bilan identitaire » (Di Méo, 2007), ce qui contribuerait à renforcer la composante cognitive du lien au territoire.

\subsection{Aspect fonctionnel}

L'aspect fonctionnel se décline en plusieurs sousaspects (culturel, spirituel, économique, santé), et ce, peu importe la génération. Il existe une pression accrue sur les plus âgés à cause de leur rôle de gardien du territoire et de transmetteur des savoirs.

La santé du territoire est directement liée à la santé de ceux qui l'utilisent (Adelson, 2004; Berkes, 2014; Parlee, Berkes et Teetl'it Gwich'in, 2005; Rigby, Rosen, Berry et Hart, 2011). La solastalgie (Albrecht, 2010) est cet état de manque, d'impuissance et de tristesse profonde qui affecte les personnes assistant quotidiennement à la lente dégradation de leur environnement, notamment par les changements climatiques et par l'exploitation des ressources naturelles. Le dysplacement (Jackson, 2011) est également un mal qui décrit un état psychologique résultant d'une détérioration de l'environnement. De ces maux découle la dépossession environnementale, qui affecte les Autochtones, dont les territoires sont de plus en plus affectés. Le développement industriel réduit l'accès aux ressources, limitant ainsi les activités économiques, sociales et culturelles qui déterminent la santé et le bien-être des communautés autochtones (Tobias et Richmond, 2014). 
Le tiers des répondants vivent de façon directe du territoire forestier puisqu'ils reçoivent des prestations du Programme de sécurité du revenu pour les chasseurs et trappeurs cris. Le choix de vivre en forêt est plus fréquent chez les Cris, probablement parce qu'il n'y a pas de programme de sécurité du revenu à Pikogan (sauf pour les Cris qui y vivent). Bien que la foresterie fournisse des emplois et des chemins d'accès au territoire, c'est également une industrie qui a des conséquences sur le mode de vie et les écosystèmes (Saint-Arnaud et collab., 2009).

Les Autochtones se considèrent gardiens du territoire (Berkes, 2014; Leroux, 2009; McGregor, Bayha et Simmons, 2010). Plus une personne vieillit, plus elle est susceptible d'hériter de la responsabilité d'un terrain de trappe. Or, les aînés sont parfois en moins bonne santé et ne peuvent se rendre en forêt aussi souvent qu'ils le voudraient (Tobias et Richmond, 2014). S'ajoutent à cela certaines contraintes logistiques telles que l'absence de moyen de transport ou d'un accompagnant, sinon la distance et les chemins forestiers fermés ou endommagés. Bien que le territoire soit le lieu privilégié de transmission des savoirs et des valeurs pour les femmes (Basile et collab., 2017), elles sont moins susceptibles de se voir transférer la responsabilité d'un terrain de trappe.

Les résultats permettent de relever un paradoxe : les aînés veulent rester dans la communauté pour des questions de santé et de bien-être, alors que les jeunes veulent partir en ville pour les mêmes raisons. Chez les aînés, leur propre état de santé ou celui d'un proche les contraint à restreindre leurs déplacements en forêt. Ils n'envisagent pas de déménager en ville puisque cela impliquerait de s'éloigner encore plus de la forêt. Pour les jeunes (18-30 ans), la ville est parfois envisagée comme un lieu d'émancipation, pour eux ou leurs enfants (Asselin et Drainville, sous presse).

Tous les répondants disent partager le fruit de leurs activités de collecte sur le territoire. En fait, avec l'eau et la médecine, la consommation de nourriture traditionnelle est le lien le plus direct entre santé et territoire (Parlee et collab., 2005; Richmond, 2015;
Simpson, DaSilva, Riffell et Sellers, 2009; Turner, Plotkin et Kuhnlein, 2013). La nourriture traditionnelle fait encore partie de l'alimentation, malgré les changements survenus dans les façons de vivre qui ont modifié les pratiques de collecte. En dépit du fait que la consommation de cette nourriture est parfois associée à certains risques (Bordeleau, Asselin, Mazerolle et Imbeau, 2016; Richmond, 2015), elle est un moyen de se connecter au territoire (Simpson et collab., 2009; Tobias et Richmond, 2014; Turner et collab., 2013).

\section{Conclusion}

Cette recherche permet de montrer que des différences existent entre les générations quant aux manifestations du lien au territoire dans deux communautés autochtones au Québec. La question se pose alors de savoir si ces différences sont tenues en compte dans les processus de prise de décision relativement au développement territorial. Des recherches supplémentaires sont nécessaires pour déterminer si le point de vue des aînés et des gestionnaires de terrains de trappe occulte celui des plus jeunes. Par ailleurs, bien que les tendances observées dans cette étude exploratoire soient claires, augmenter la taille de l'échantillon et le nombre de communautés permettrait d'explorer statistiquement les relations complexes entre les trois aspects du lien au territoire.

Les résultats de cette recherche soulèvent des questions qui pourraient être abordées dans des projets futurs. Par exemple, puisque la vitalité des langues autochtones semble étroitement liée à l'utilisation du territoire, il conviendrait d'étudier les effets des changements environnementaux sur la transmission linguistique. De plus, puisque le soutien familial est un élément fondamental du lien au territoire, plus de recherches sont nécessaires pour identifier les façons de favoriser la solidarité intergénérationnelle. Enfin, considérant que la ruralité autochtone ne se vit pas en vase clos et implique des déplacements fréquents entre la forêt, la communauté et la ville, de futures études pourraient se pencher sur les conditions favorisant la mobilité.

\section{REMERCIEMENTS}

Les auteurs remercient sincèrement tous les répondants pour leur générosité. Maggie Etapp, Dennis Saganash et Allan Saganash (Waswanipi), ainsi que James Cananasso, Roxane Germain et Benoît Croteau (Pikogan) ont grandement facilité la collecte de données. Cette recherche a été financée par le Conseil de recherche en sciences humaines du Canada, par le Programme de formation scientifique dans le Nord et par le Réseau de recherche et de connaissances relatives aux peuples autochtones (DLALOG). 


\section{NOTES}

1 La majorité des personnes âgées sont considérées comme des aînés en raison de leur expérience. Toutefois, il ne suffit pas d'être âgé pour être considéré ainé. Dans cette recherche, les personnes de 61 ans et plus sont nommées « ainés » par souci de simplicité.

\section{RÉFÉRENCES}

Adelson, N. (2004). «Being alive well »: Health and the politics of Cree well-being. Toronto, ON: University of Toronto Press.

Albrecht, G. (2010). Solastalgia and the creation of new ways of living. Dans J. Pretty et S. Pilgrim (dir.), Nature and culture: Rebuilding lost connections (p. 217-234). Londres, R.-U.: Earthscan.

Anton, C. E. et Lawrence, C. (2014). Home is where the heart is: The effect of place of residence on place attachment and community participation. Journal of Environmental Psychology, 40, 451-461. https://doi.org/10.1016/j.jenvp.2014.10.007

Ashmore, R. D., Deaux, K. et McLaughlin-Volpe, T. (2004). An organizing framework for collective identity: Articulation and significance of multidimensionality. Psychological Bulletin, 130(1), 80-114. https://doi.org/10.1037/0033-2909.130.1.80

Asselin, H. (2015). Indigenous forest knowledge. Dans K. Peh, R. Corlett et Y. Bergeron (dir.), Routledge handbook of forest ecology (p. 586-596). New York, NY: Routledge.

Asselin, H. et Drainville, R. (2020). Are Indigenous youth in a tug-of-war between community and city? Reflections from a visioning workshop in the Lac Simon Anishnaabeg community (Quebec, Canada). World Development Perspectives. 17, 100168 https://doi.org/10.1016/j.wdp.2019.100168

Barreau, A., Ibarra, J. T., Wyndham, F. S., Rojas, A. et Kozak, R. A. (2016). How can we teach our children if we cannot access the forest? Generational change in Mapuche knowledge of wild edible plants in Andean temperate ecosystems of Chile. Journal of Ethnobiology, 36(2), 412-432. https://doi.org/10.2993/0278-0771-36.2.412

Basile, S., Asselin, H. et Martin, T. (2017). Le territoire comme lieu privilégié de transmission des savoirs et des valeurs des femmes Atikamekw. Recherches féministes, 30(1), 61-80. https://doi.org/10.7202/1040975ar

Berkes, F. (2014). Sacred ecology. New York, NY: Routledge.

Berry, H., Butler, J., Burgess, C., King, U., Tsey, K., Cadet-James, Y. L., Wayne Rigby, C. et Raphael, B. (2010). Mind, body, spirit: Co-benefits for mental health from climate change adaptation and caring for country in remote Aboriginal communities. New South Wales Public Health Bulletin, 21(6), 139-145. https://doi.org/10.1071/NB10030

Big-Canoe, K. et Richmond, C. A. (2014). Anishinabe youth perceptions about community health: Toward environmental repossession. Health \& Place, 26, 127-135. https://doi.org/10.1016/j.healthplace.2013.12.013

Bordeleau, S., Asselin, H., Mazerolle, M. J. et Imbeau, L. (2016). « Is it still safe to eat traditional food? » Addressing traditional food safety concerns in aboriginal communities. Science of the Total Environment, 565, 529-538. https://doi.org/10.1016/ j.scitotenv.2016.04.189

Bousquet, M.-P. (2002). "Quand nous vivions dans le bois 》, le changement spatial et sa dimension générationnelle : l'exemple des Algonquins du Canada (thèse de doctorat). Université de Paris X-Nanterre et Université Laval, Paris (France) et Québec (Canada).

Bousquet, M.-P. (2005). Les jeunes Algonquins sont-ils biculturels? Recherches amérindiennes an Québec, 35(3), 7-17.

Cheng, A. S., Kruger, L. E. et Daniels, S. E. (2003). «Place» as an integrating concept in natural resource politics: Propositions for a social research agenda. Society and Natural Resources, 16(2), 87-104. https://doi.org/10.1080/08941920309199

Desbiens, C., Lévesque, C. et Comat, I. (2016). «Inventing new places »: Urban aboriginal visibility and the co-construction of citizenship in Val-d’Or (Québec). City \& Society, 28(1), 74-98. https://doi.org/10.1111/ciso.12074

Dietsch, E., Martin, T., Shackleton, P., Davies, C., McLeod, M. et Alston, M. (2011). Australian Aboriginal kinship: A means to enhance maternal well-being. Women and Birth, 24(2), 58-64. https://doi.org/10.1016/j.wombi.2010.06.003

Di Méo, G. (2007). Identités et territoires : des rapports accentués en milieu urbain? Métropoles, 1, 69-94. https://doi.org/10.4000/metropoles. 80

Fuentes, L., Asselin, H., Bélisle, A. C. et Labra, O. (2020). Impacts of environmental changes on well-being in Indigenous communities in eastern Canada. International Journal of Environmental Research and Public Health, 17(2), 637. https://doi.org/10.3390/ijerph17020637 
Gamborg, C., Parsons, R., Puri, R. K. et Sandøe, P. (2012). Ethics and research methodologies for the study of traditional forestrelated knowledge. Dans J. A. Parrotta et R. L. Trosper (dir.), Traditional forest-related knowledge: Sustaining communities, ecosystems and biocultural diversity (p. 535-562). Dordrecht, Netherlands: Springer.

Germain, R. (2012). Acceptabilité sociale de l'aménagement forestier écosystémique : le point de vue des Algonquins de Pikogan (mémoire de maitrise). Université du Québec en Abitibi-Témiscamingue, Rouyn-Noranda, Canada.

Guérin-Pace, F. (2006). Lieux habités, lieux investis : le lien au territoire, une composante identitaire? Économie et statistique, 393-394, 101-114. https://doi.org/10.3406/estat.2006.7144

Hernández, B., Hidalgo, M. C., Salazar-Laplace, M. E. et Hess, S. (2007). Place attachment and place identity in natives and nonnatives. Journal of Environmental Psychology, 27(4), 310-319. https://doi.org/10.1016/j.jenvp.2007.06.003

Jackson, D. D. (2011). Scents of place: The dysplacement of a First Nations community in Canada. American Anthropologist, 113(4), 606-118. https://doi.org/10.1111/j.1548-1433.2011.01373.x

Jean, B. (2017). Les ruraux comme minorité invisible, mais agissante : propos sur la construction sociale de la ruralité. Dans G. Chiasson, A. Ependa, B. Jean et P. LeBlanc (dir.), Actes du colloque «L L ruralité au Québec depuis les États généraux du monde rural (1991) : entre l'action et la recherche, bilan et perspectives» (p. 176-182). Montréal, QC : Association francophone pour le savoir.

Jorgensen, B. S. et Stedman, C. R. (2006). A comparative analysis of predictors of sense of place dimensions: Attachment to, dependence on, and identification with lakeshore properties. Journal of Environmental Management, 79(3), 316-327. https://doi.org/10.1016/j.jenvman.2005.08.003

Junot, A., Paquet, Y. et Fenouillet, F. (2018). Place attachment influence on human well-being and general pro-environmental behaviors. Journal of Theorical Social Psychology, 2(2), 49-57. https://doi.org/10.1002/jts5.18

Kermoal, N. et Lévesque, C. (2010). Repenser le rapport à la ville : pour une histoire autochtone de l'urbanité. Nowvelles pratiques sociales, 23(1), 67-82. https://doi.org/10.7202/1003168ar

Kistabish, B., Kistabish, D., Mapachee, T. et Rankin, S. (2011). «On est les éternels oubliés » : les Abitibiwinniks de Pikogan. Recherches amérindiennes an Québec, 41(1), 67-69. https://doi.org/10.7202/1012705ar

Landry, V., Asselin, H. et Lévesque, C. (2019). Link to the land and mino-pimatisiwin (comprehensive health) of Indigenous people living in urban areas in eastern Canada. International Journal of Environmental Research and Public Health, 16(23), 4782. https://doi.org/10.3390/ijerph16234782

Lannegrand-Willems, L. (2012). Le développement de l'identité à l'adolescence : quels apports des domaines vocationnels et professionnels? Enfance, 3(3), 313-327. https://doi.org/10.4074/S0013754512003060

Lemelin, H., Matthews, D., Mattina, C., McIntyre, N., Johnston, M. et Koster, R. (2010). Climate change, well-being, and resilience in the Weenusk First Nation at Peawanuck: The Moccasin Telegraph goes global. Rural and Remote Health, 10, 1333.

Leroux, J. (2009). Éthique et symbolique de la responsabilité territoriale chez les peuples algonquiens du Québec. Recherches amérindiennes an Québec, 39(1-2), 85-97. https://doi.org/10.7202/044999ar

Lévesque, C. et Cloutier, É. (2013). Les Premiers Peuples dans l'espace urbain au Québec : trajectoires plurielles. Dans A. Beaulieu, M. Papillon et S. Gervais (dir.), Les Autochtones et le Québec (p. 281-296). Montréal, QC : Presses de l'Université de Montréal.

Low, S. M. et Altman, I. (1992). Place attachment. Dans I. Altman et S. M. Low (dir.), Place attachment: Human behavior and environment, advances in theory and research (vol. 12, p. 1-12). Boston, MA : Springer.

Martin, T. et Girard, A. (2009). Le territoire « matrice de culture » : analyse des mémoires déposés à la commission Coulombe par les premières nations du Québec. Recherches amérindiennes au Québec, 39(1-2), 61-69. https://doi.org/10.7202/044997ar

McGrath, P. (2007). «I don’t want to be in that big city; this is my country here»: Research findings on Aboriginal peoples’ preference to die at home. Australian Journal of Rural Health, 15(4), 264-268. https://doi.org/10.1111/j.1440-1584.2007.00904.x

McGregor, D., Bayha, W. et Simmons, D. (2010). « Our responsability to keep the land alive »: Voices of northern Indigenous researchers. Pimatisiwin: A Journal of Aboriginal and Indigenous Community Health, 8(1), 101-123.

McMillan, D. W. et Chavis, D. M. (1986). Sense of community: A definition and theory. Journal of Community Psychology, 14(1), 6-23. https://doi.org/10.1002/1520-6629(198601)14:1<6::AID-JCOP2290140103>3.0.CO;2-I

Niezen, R. (2016). Defending the land: Sovereignty and forest life in James Bay Cree society. New York, NY: Routledge. 
Parlee, B., Berkes, F. et Teetl'it Gwich'in. (2005). Health of the land, health of the people: A case study on Gwich'in berry harvesting in Northern Canada. EcoHealth, 2(2), 127-137. https://doi.org/10.1007/s10393-005-3870-z

Ramos, E. (2006). L'invention des origines : sociologie des ancrages identitaires. Paris, France : Armand Colin.

Richmond, C. (2015). The relatedness of people, land and health: Stories from Anishnabe elders. Dans M. Greenwood, S. De Leeuw, N. M. Lindsay et C. Reading (dir.), Determinants of Indigenous peoples' health in Canada: Beyond the social. Toronto, ON: Canadian Scholars' Press.

Richmond, C. A. et Ross, N. A. (2009). The determinants of First Nation and Inuit health: A critical population health approach. Health \& Place, 15(2), 403-411. https://doi.org/10.1016/j.healthplace.2008.07.004

Rigby, C. W., Rosen, A., Berry, H. L. et Hart, C. R. (2011). If the land's sick, we're sick: The impact of prolonged drought on the social and emotional well-being of Aboriginal communities in rural New South Wales. Australian Journal of Rural Health, 19(5), 249-254. https://doi.org/10.1111/j.1440-1584.2011.01223.x

Saint-Arnaud, M., Asselin, H., Dubé, C., Croteau, Y. et Papatie, C. (2009). Developing criteria and indicators for aboriginal forestry: Mutual learning through collaborative research. Dans M. G. Stevenson et D. C. Natcher (dir.), Changing the culture of forestry in Canada: Building effective institutions for Aboriginal engagement in sustainable forest management (p. 85-105). Edmonton, AB : Canadian Circumpolar Institute Press.

Scannell, L. et Gifford, R. (2010). The relations between natural and civic place attachment and pro-environmental behavior. Journal of Environmental Psychology, 30(3), 289-297. https://doi.org/10.1016/j.jenvp.2010.01.010

Simpson, L., DaSilva, J., Riffell, B. et Sellers, P. (2009). The responsibilities of women: Confronting environmental contamination in the traditional territories of Asubpeechoseewagong Netum Anishinabek (Grassy Narrows) and Wabauskang First Nation. Journal of Aboriginal Health, 4(2), 6-13. https://doi.org/10.3138/ijih.v4i2.28968

Stedman, R. C. (2003). Sense of place and forest science: Toward a program of quantitative research. Forest Science, 49(6), $822-829$. https://doi.org/10.1093/forestscience/49.6.822

Tidball, K. et Stedman, R. (2013). Positive dependency and virtuous cycles: From resource dependence to resilience in urban social-ecological systems. Ecological Economics, 86, 292-299. https://doi.org/10.1016/j.ecolecon.2012.10.004

Tobias, J. K. et Richmond, C. A. (2014). «That land means everything to us as Anishinaabe....»: Environmental dispossession and resilience on the North Shore of Lake Superior. Health \& Place, 29, 26-33. https://doi.org/10.1016/j.healthplace.2014.05.008

Turner, N. J., Plotkin, M. et Kuhnlein, H. V. (2013). Global environmental challenges to the integrity of Indigenous peoples' food systems. Dans H. V. Kuhnlein, B. Erasmus, D. Spigelski et B. Burlingame (dir.), Indigenous peoples' food systems \& well-being: Interventions \& policies for healtby communities (p. 23-38). Rome, Italie : Organisation des Nations unies pour l'alimentation et l'agriculture.

Viscogliosi, C., Asselin, H., Basile, S., Borwick, K., Couturier, Y., Drolet, M.-J., Gagnon, D., Obradovic, N., Torrie, J., Zhou, D. et Levasseur, M. (sous presse). Importance of Indigenous elders' contributions to individual and community wellness: Results from a scoping review on social participation and intergenerational solidarity. Canadian Journal of Public Health. https://doi.org/10.17269/s41997-019-00292-3.

Wilson, K. (2003). Therapeutic landscapes and First Nations peoples: An exploration of culture, health and place. Health \& Place, 9(2), 83-93. https://doi.org/10.1016/S1353-8292(02)00016-3

Wilson, K., Rosenberg, W. M. et Abonyi, S. (2011). Aboriginal peoples, health and healing approaches: The effects of age and place on health. Social Science \& Medicine, 72(3), 355-364. https://doi.org/10.1016/j.socscimed.2010.09.022 